\title{
New Training Approach for Improving the Spatial Perception and Orientation Ability of Dentistry Students
}

\author{
Asaf Shely ${ }^{1, *}++^{\mathbb{D}}$, Gil Ben-Izhack ${ }^{1,+} \mathbb{D}$, Diva Lugassy ${ }^{2}$, Shlomo Matalon ${ }^{1} \mathbb{D}$, Raphael Pilo ${ }^{2} \mathbb{D}$, Tamar Brosh $^{2,+}$ \\ and Eran Dolev ${ }^{1,+}$
}

1 Department of Oral Rehabilitation, The Maurice and Gabriela Goldschleger School of Dental Medicine, Sackler Faculty of Medicine, Tel Aviv University, Tel Aviv 6997801, Israel; gil.ben.izhack@gmail.com (G.B.-I.); matalons@tauex.tau.ac.il (S.M.); eran@drdolev.com (E.D.)

2 Department of Oral Biology, The Maurice and Gabriela Goldschleger School of Dental Medicine, Sackler Faculty of Medicine, Tel Aviv University, Tel Aviv 6997801, Israel; diva_lugassy@hotmail.com (D.L.); rafipilo@gmail.com (R.P.); tbrosh@tauex.tau.ac.il (T.B.)

* Correspondence: asafshely@gmail.com; Tel.: +972-528787472

+ These authors contributed equally to the work.

Citation: Shely, A.; Ben-Izhack, G.; Lugassy, D.; Matalon, S.; Pilo, R.; Brosh, T.; Dolev, E. New Training Approach for Improving the Spatial Perception and Orientation Ability of Dentistry Students. Appl. Sci. 2021, 11, 9387. https://doi.org/10.3390/ app11209387

Academic Editor: Oleh Andrukhov

Received: 9 August 2021

Accepted: 6 October 2021

Published: 9 October 2021

Publisher's Note: MDPI stays neutral with regard to jurisdictional claims in published maps and institutional affiliations.

Copyright: (c) 2021 by the authors. Licensee MDPI, Basel, Switzerland. This article is an open access article distributed under the terms and conditions of the Creative Commons Attribution (CC BY) license (https:// creativecommons.org/licenses/by/ $4.0 /)$.

\begin{abstract}
Introduction: During a basic prosthetics course, dental students train on plastic teeth for fixed partial dentures (FPD). The complexity of manual skill acquisition and the need for extra training led us to develop a portable tool for home training (PhantHome). The aim of the current study was to assess whether training using the portable tool improves students fine motor skill, spatial perception, and orientation and may predict success in preclinical prosthetics courses. Material and Methods: A total of 42 third year dental students were included in this study. A valid dexterity test (Grooved Pegboard test) and a manual test using the portable tool (PhantHome) were conducted in direct and indirect visions using a mirror at two time points: T0: beginning of study, and T1: after training for one month with the portable tool at home. The students' manual grades in the portable tool, Grooved test, and final prosthetics course grades were compared. Results: The results showed that indirect tasks were significantly more difficult to perform than direct tasks for PhantHome and Grooved tests at T0 and T1 $(p<0.0005)$. After practicing with the portable PhantHome tool (T1), the students' scores of in PhantHome and Grooved tests improved significantly $(p<0.04)$. A regression analysis showed that students' motor tasks scored at T0 predicted phantom course success in $86.8 \%$ of cases $(p=0.005)$. Conclusion: There was a positive transfer in learning: PhantHome training led to improved performance on the Grooved tests without further training on these tests. Therefore, training in the PhantHome tool can significantly improve performance in the prosthodontics phantom course. The prediction model predicted success in a prosthodontics course with $86 \%$ accuracy.
\end{abstract}

Keywords: dental student; manual skills; PhantHome; Grooved Pegboard Test

\section{Introduction}

During a pre-clinical prosthetics course, dental students learn the principles of performing dental preparations for fixed partial dentures (FPD). One of the major problems students face in the course is spatial perception and orientation, which can be manifested in poor identification of the tooth axis and difficulty in identifying undercuts in the preparation, leading to inadequate preparation. Furthermore, the difficulty arises when assignments require indirect vision using a dental mirror, which requires manual skills for working with both hands. [1,2]. Learning this skill is considered difficult due to the natural difficulty of the brain to control two new complex motor actions that require coordination of both hands.

Tooth preparation for FPD is a clinical challenge for students because the designs must meet important biomechanical principles in order to meet accepted standards to receive a permanent crown [3]. Two of the basic principles in preparing for FPD are the principle 
of resistance and retention. One of the goals of retention is to have a single insertion path that is parallel to the tooth's longitudinal axis. The insertion path is determined by tooth preparation, and tilting of the opposite walls properly is critical to the retention of the preparation. Optimally, we would like the insertion path to be parallel to the tooth's longitudinal axis, so that it should always be $90^{\circ}$ to the occlusal surface and inclined at a convergence angle of 6-12 ${ }^{\circ}$ [4-6]. Researchers found that dental students performed on averaged 12.7 degrees of wall convergence, while the clinical preparations average was 22.8 degrees $[7,8]$. Another study that modeled 909 dental preparations for FPD found that the average convergence of preparations was 19.2 degrees [9].

In order to increase the accuracy of dental students' preparations, researchers have developed innovative digital design software for helping students understand the tooth's axis, marginal design, and undercuts, and objectively self-evaluate their preparation [10-12]. For example, PrepCheck (Dentsply Sirona, Wien, Austria) makes use of CAD/CAM (computerassisted design and computer-assisted manufacturing) technology by comparing scans of tooth preparations with a standardized "master" preparation [13]. Although accumulating evidence has confirmed the effectiveness of such new assessment methods, primarily in relation to self-assessment and self-directed learning of manual skills, most studies have claimed that further investigation of CAD/CAM technology for the purpose of assessment and education of dental students is recommended [14,15].

Other researchers believe that manual performance can be facilitated by means of a positive transfer of learning. Accordingly, repeated training of one motoric task leads to significant improvement in the performance of another motoric task that has similar components [16]. They developed training tools to enhance performance in preclinical courses. For example, Gunter et al. developed an indirect training device called Mirroprep, which consists of a high-profile quality steel sheet with a mirror mounted to its rearmost wall and a replica of a dental drill holding a short pencil. The test sheet consists of a curved track course in different outline forms, in which the student must track the course on the pad bounded by two lines with the pencil. They showed that mirror vision can be learned and improved while practicing on the Mirroprep; the result was a high degree of transfer in manual performance $[17,18]$. The disadvantage of training tools that have been developed is that the actual conditions of the training were not similar enough to the phantom laboratory environment.

In this study, a simple portable manual training tool was developed for training at home that entails the general principles and identical elements as in the pre-clinical prosthetics course and can be used as a supplement to conventional training as needed. In addition, the novel tool may serve to screen dentistry candidates and to evaluate their manual capabilities. The tool comprises components that are very similar, but not identical, to the traditional phantom head simulator and allow training for improving fine motor skill, spatial perception, and orientation, capabilities that are required in order to performed acceptable tooth preparation for crowns. The tool includes plastic teeth with five drills at a $6^{\circ}$ angle, corresponding to an acceptable angle of convergence of tooth preparations. The students have to insert pins into these drills under direct and indirect vision for training. As an evaluation tool of students' capabilities, the Grooved pegboard test, a valid and reliable test from the occupational therapy field that measures eye-hand coordination, manual skills, and orientation by insertion of pins (key-shaped) into grooves with different angles, was adopted [19-21].

Thus, it was hypothesized that: (1) practice at home on the portable tool would lead to a significant improvement in fine motor skills, spatial perception, and orientation, and (2) the portable tool may predict the potential success of dental students in a preclinical prosthetics course. Therefore, the aim of the current study was to assess whether training using the portable tool improves students' fine motor skills, spatial perception, and orientation and may predict success in preclinical prosthetics courses. 


\section{Materials and Methods}

Study Population

Forty-two dental students in their third year of study at the School of Dental Medicine at Tel Aviv University participated in the study. Of these, 31 were women and 11 were men, aged between 22 and 36 years (mean $26 \pm 3.09$ years). The inclusion criteria were generally healthy, normal, or corrected vision; right- or left-hand dominance; not having participated in a dental morphology course; and not yet having been trained in manual skills courses at the simulation phantom laboratory. The study was conducted according to the guidelines and approved by the Institutional Ethics Committee of Tel Aviv University (16 September 2018), and all participants signed an informed consent form. The research exclusion criteria were students with a health problem, students who have had a dominant palm injury for the past two years, or students who have suffered from skeletal muscle pain within the last month. At the beginning of the study, each student completed a questionnaire that included demographic (age, gender) and questions related to components that could affect the student's motor performance, for example, leisure activities and hobbies involving gross and delicate motor skills.

\section{Protocols}

Two different tools were utilized in this study: (1) the Grooved Pegboard test modification, and (2) the PhantHome training tool. The students performed both tests twice: at the beginning of study (T0) and after one month (T1) of practicing at home with the PhantHome tool.

\subsection{Grooved Pegboard Test}

This test is used to measure hand-eye coordination, manual skills, and orientation [21-24]. The test is conducted using a board with 25 slots in the shape of a keyhole, located at random angles. In order to insert the pins into the groove, they must be rotated like a key corresponding to the groove. Modification to the test was carried out to allow for performance of the test in indirect vision. This was obtained by hiding the task-board by a black screen and observing the task through a mirror. Each participant first performed the test with direct vision using the dominant hand followed by his/her non-dominant hands, and then indirectly in a similar order. The time taken for the completion of each of the four tests was the score for the test (Figure 1).

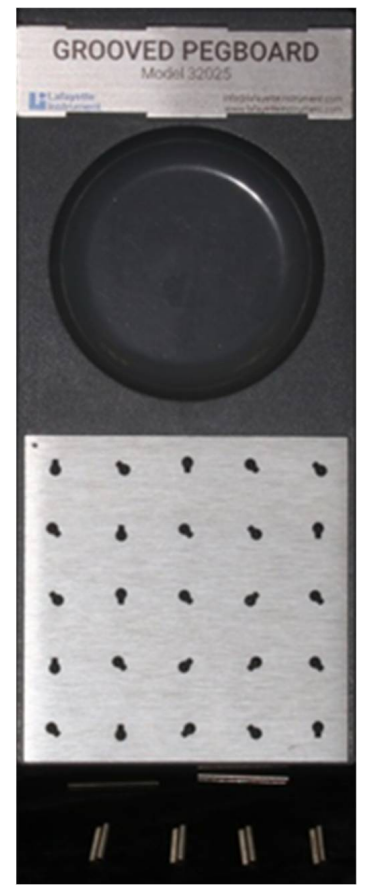

Figure 1. The Grooved Pegboard test device. 


\subsection{Innovative Portable Training Tool: PhantHome}

The portable interventional tool is composed of a commercial phantom head composed of two jaws with plastic teeth, artificial gingival tissue, and a rubber cover, which simulate the mouth of the patient (Nissin Dental Products INC, Nakagoku, Japan). The first molar tooth in each quadrant was replaced with special plastic teeth with five holes at a depth of $4 \mathrm{~mm}$, a central hole along the tooth axis at 90 degrees to the occlusal surface, and four holes converging to a central hole at a 6-degree angle corresponding to the dental preparations (Figure 2).

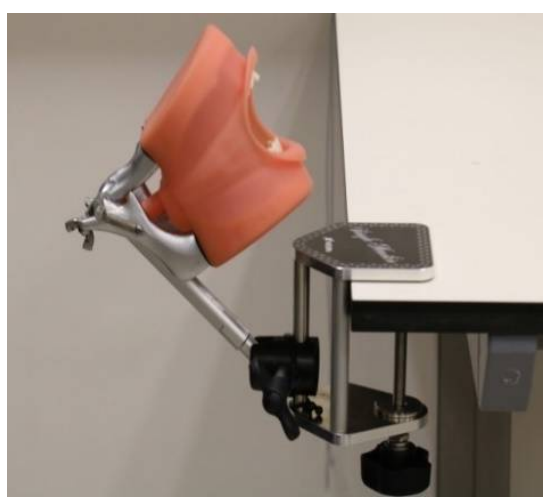

(A)

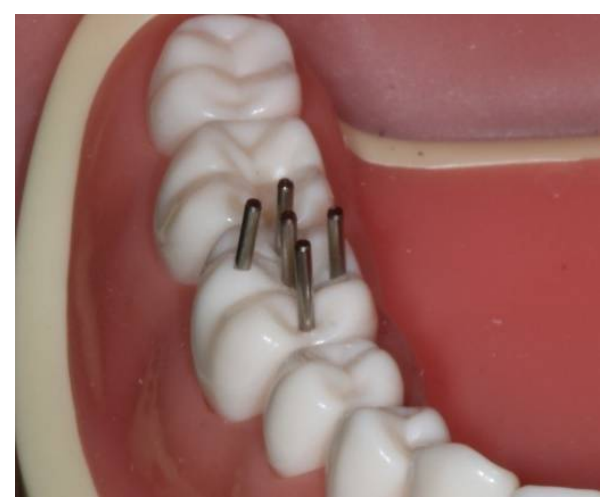

(B)

Figure 2. (A). Mobile device, desktop fixation, silicone simulating soft tissue. (B). Close view: tooth 36 with all 5 pins.

The students were instructed to insert matching metal pins $(1 \mathrm{~mm}$ diameter, $10 \mathrm{~mm}$ length) in a specific order into these holes. The PhantHome tool can be attached to any table with a compatible stand. All the components of the kit are organized in a small carrying box. Each student at the beginning of the study was given a home training kit for one month. Students were given practice sheets and were instructed to practice at least twice a week in all exercises. The training included using both hands: the dominant hand for inserting pins using tweezers and while working in the upper jaw, and the student was required to use a dental mirror with his/her non-dominant hand in indirect vision. The insertion of the pins in the home exercise was performed on four teeth: the upper and lower first molars. The variable measured when performing the test on PhantHome was minutes needed to perform the 20-pin insertion task (five pins per tooth, four teeth).

\subsection{Study Procedure}

The experiment was performed at two time points, before training with the portable tool (T0) and after training (T1).

During each period, all students performed the same order of testing: four tasks on the Grooved Pegboard test (direct dominant hand, direct non-dominant hand, indirect dominant hand, and indirect non-dominant hand) and two tests on the PhantHome tool under direct and indirect vision. The direct test included insertion of 10 pins into the lower first molars by direct vision with tweezer ( 5 pins per tooth). The indirect test included insertion of 10 pins into the upper first molars by indirect vision using tweezers and a dental mirror.

The time necessary to administer the battery of all tests during each period ranged from 20 to 30 min per participant.

Students' dental performance was assessed at the end of the prosthodontics course at a phantom laboratory via the final phantom grade. The final phantom grade (Final.Phantom) was the average grade of the three tasks routinely provided by the clinical instructors at the end of the prosthodontic dentistry course performed on plastic that included anterior tooth preparation for FPD, posterior tooth preparation for FPD, and a temporary acrylic crown. Three instructors provided the grades using several common criteria (evaluation form), 
such as inclination of tooth preparation, long axis of the preparation, and other criteria (see evaluation form, Appendix A). The grades are on a continuous scale of $0-100$, where the passing grade is 60 . As the student identification numbers were coded, the instructors were unaware of the individual students' results. The study was blinded in the sense that the principal investigator who conducted the dexterity tests was blind to the students' final grades for the phantom course.

\subsection{Statistics}

Students' scores were defined by the time (in minutes) to perform each task.

The assumptions of the normality of the variables (students' scores on the Grooved tests and PhantHome and students' grades on the prosthodontics course) were assessed using the Kolmogorov-Smirnov test. For comparisons between the test scores in the Grooved Pegboard test and PhantHome tests with direct and indirect vision and between $\mathrm{T} 0$ and $\mathrm{T} 1$, Wilcoxon signed ranks tests were performed.

Kendall's nonparametric correlations were calculated between the two manual dexterity tests at T0: the Grooved Pegboard test and PhantHome Test and between the final grades in the prosthodontics course. A linear regression model using the enter method, as well as a logistic regression using the enter method, were conducted to examine the strength and combination of the explanatory variables of the final grade in prosthodontics course. All analyses were performed using SPSS version 20. Significant statistical differences were defined as $p<0.05$.

\section{Results}

Before the data were analyzed, the Kolmogorov-Smirnov tests were performed on students' scores of the Grooved tests and PhantHome and students' grades of the prosthodontics course, which indicated a no normal distribution for the variables (grades) $(p<0.05)$.

The scores of the students on the Grooved Pegboard test for both hands under indirect vision were significantly higher than those under direct vision $(p<0.005)$ across the two time periods (Table 1). Similar trends were observed for the PhantHome test in which the scores under indirect vision were significantly higher than those under direct vision $(p<0.005)$ across the two time periods (note that the scores on both tests represent the time needed to insert pins).

Table 1. The mean, standard deviation (SD), and range of the Grooved Pegboard test and PhantHome test scores under direct and indirect vision conditions at the two time periods $(\mathrm{T} 0, \mathrm{~T} 1)$ for dental students $(\mathrm{N}=42)$.

\begin{tabular}{ccccc}
\hline Motor Task & \multicolumn{3}{c}{ T0 } & \multicolumn{2}{c}{ T1 } \\
\cline { 2 - 5 } & Mean $( \pm$ SD) & [Range] (min) & Mean ( \pm SD) & [Range] (min) \\
\hline Grooved Pegboard_Direct_Dominant Hand & $1( \pm 0.16)$ & {$[0.43-1.36]$} & $0.9( \pm 0.1)$ & {$[0.53-1.25]$} \\
\hline Grooved Pegboard_Direct_Non Dominant Hand & $1.1( \pm 0.17)$ & {$[0.8-1.65]$} & $1(0.18)$ & {$[0.75-1.6]$} \\
\hline Grooved Pegboard_Indirect_Dominant Hand & $3.6( \pm 1.6)$ & {$[1.83-7.6]$} & $2.4( \pm 0.9)$ & {$[1.45-5.46]$} \\
\hline Grooved Pegboard_Indirect_Non Dominant Hand & $3.9( \pm 1.7)$ & {$[1.7-9.9]$} & $2.9( \pm 1.1)$ & {$[1.46-7.18]$} \\
\hline PhantHome_Direct & $2.6( \pm 2.3)$ & {$[0.6-13.8]$} & $1.6( \pm 0.88)$ & {$[0.68-5]$} \\
\hline PhantHome_Indirect & $3.9( \pm 3)$ & {$[0.7-12.7]$} & $2.4( \pm 1.3)$ & {$[0.76-8.33]$} \\
\hline
\end{tabular}

After practicing with the portable PhantHome tool (T1), the students' scores significantly decreased for all tasks under direct and indirect vision on the Grooved Pegboard test and PhantHome test. The level of performance in the PhantHome test improved significantly under direct $(p=0.01)$ and indirect vision $(p=0.004)$. Interestingly, the level of performance in the Grooved Pegboard test also improved significantly under direct vision in the dominant hand $(p=0.005)$ and in the non-dominant hand $(p=0.04)$, and under indirect vision in the dominant hand $(p=0.005)$ and the non-dominant hand $(p=0.005)$. We found a positive transfer between training in the PhantHome tool and the result in 
the Grooved tests, meaning that repeated training of one motoric task leads to significant improvement in the performance of another motoric task that has similar components.

To test the strength of the association between the manual/clinical tests performed at the end of the prosthodontics course and the motor tests in the Grooved Pegboard test and PhantHome at T0, we performed Kendall's correlation tests (Table 2). Interestingly, there was an inverse relationship between most of the motoric tests at the beginning of the study (T0), which tested orientation and spatial perception by inserting pins in different directions into the test panel and the degree of success in the final exam of the prosthodontics course; that is, the shorter the time in which the motor task was performed, the higher the final scores. It was noted that the highest correlation coefficient (moderate correlation) was found in the PhantHome test under direct vision $(r=-0.379, p=0.019)$.

Table 2. Correlations between prosthodontic final grade and motor tests performed at time T0.

\begin{tabular}{cc}
\hline Motor Task & Final Grade in Prosthodontics Course \\
\hline Grooved Pegboard_Direct_Dominant hand & -0.290 \\
Grooved Pegboard_Direct_Non-Dominant hand & $p=0.012$ \\
\hline Grooved Pegboard t_Indirect_Dominant hand & -0.288 \\
& $p=0.013$ \\
\hline Grooved Pegboard t_Indirect_Non Dominanat hand & -0.124 \\
& $p=0.274$ \\
\hline PhantHome_Direct & -0.316 \\
\hline \multirow{2}{*}{ PhantHome_Indirect } & $p=0.005$ \\
\hline
\end{tabular}

Furthermore, a linear regression analysis using the enter variable selection method using all independent variables that revealed significant correlations in the previous Kendall's correlation tests was applied. All independent variables explained $37 \%$ of the variance in the final grade of the prosthodontics course $\left(R^{2}=0.37\right)$ and produced a significant model $(p<0.003)$.

The students were categorized on the basis of their success or failure in the prosthodontics course (a passing grade was defined as more than 60 of 100 points). A logistic regression analysis was conducted using the four predictor independent variables that showed significance correlations in the linear regression test (Grooved Pegboard Test_Direct_Dominant, Grooved Pegboard Test_Direct_Non-Dominant hand, Grooved Pegboard Test_Indirect_Non Dominanat hand, and PhantHome_Direct hand) on the final prosthodontic grade (predicted dependent variable). The model was significant $(p=0.005)$ and led to correct predictions in $86.8 \%$ of the cases; in other words, $13.2 \%$ of the successes or failures were erroneously classified oppositely (Table 3). The odds ratios, using the Enter method for that test, are presented in Table 4. The test shows that the manual tests for predicting success in prosthodontic course are Grooved Pegboard Test_Indirect_Non Dominant and PhantHome_Direct hand. Classifying the final grades results to quartiles, we found that the Grooved Pegboard Test was sensitive to addressing the weak students (Figure 3). This will be discussed later. 
Table 3. Logistic regression model for predicting the dependent variable and final grade in the rehabilitation course.

\begin{tabular}{cccc}
\hline \multirow{2}{*}{ Observed } & \multicolumn{3}{c}{ Predicted } \\
\cline { 2 - 4 } & Failed & Passed & Percentage of Predictability \\
\hline Failed & 10 & 4 & $71.4 \%$ \\
\hline Passed & 1 & 23 & $95.8 \%$ \\
\hline $\begin{array}{c}\text { Overall percentage of } \\
\text { prediction model readiness }\end{array}$ & & $86.8 \%$ \\
\hline
\end{tabular}

Table 4. Logistic regression analysis for explanatory variables to predict the final grade in the prosthodontic course using the Enter method.

\begin{tabular}{ccccccc}
\hline Motor Task & B & Sig & Exp(B) & \multicolumn{2}{c}{ 95\% C.I. for Exp(B) } \\
\hline & & & & Lower & Upper \\
\hline Grooved Pegboard Test_Direct_Dominant & -4.473 & 0.125 & 0.011 & 0.005 & 3.461 \\
\hline Grooved Pegboard Test_Direct_Non-Dominant hand & -2.257 & 0.455 & 0.105 & 0.005 & 39.154 \\
\hline Grooved Pegboard Test_Indirect_Non Dominanat & -0.652 & 0.044 & 0.521 & 0.267 & 0.983 \\
\hline PhantHome_Direct hand & -0.515 & 0.05 & 0.598 & 0.357 & 1 \\
\hline
\end{tabular}

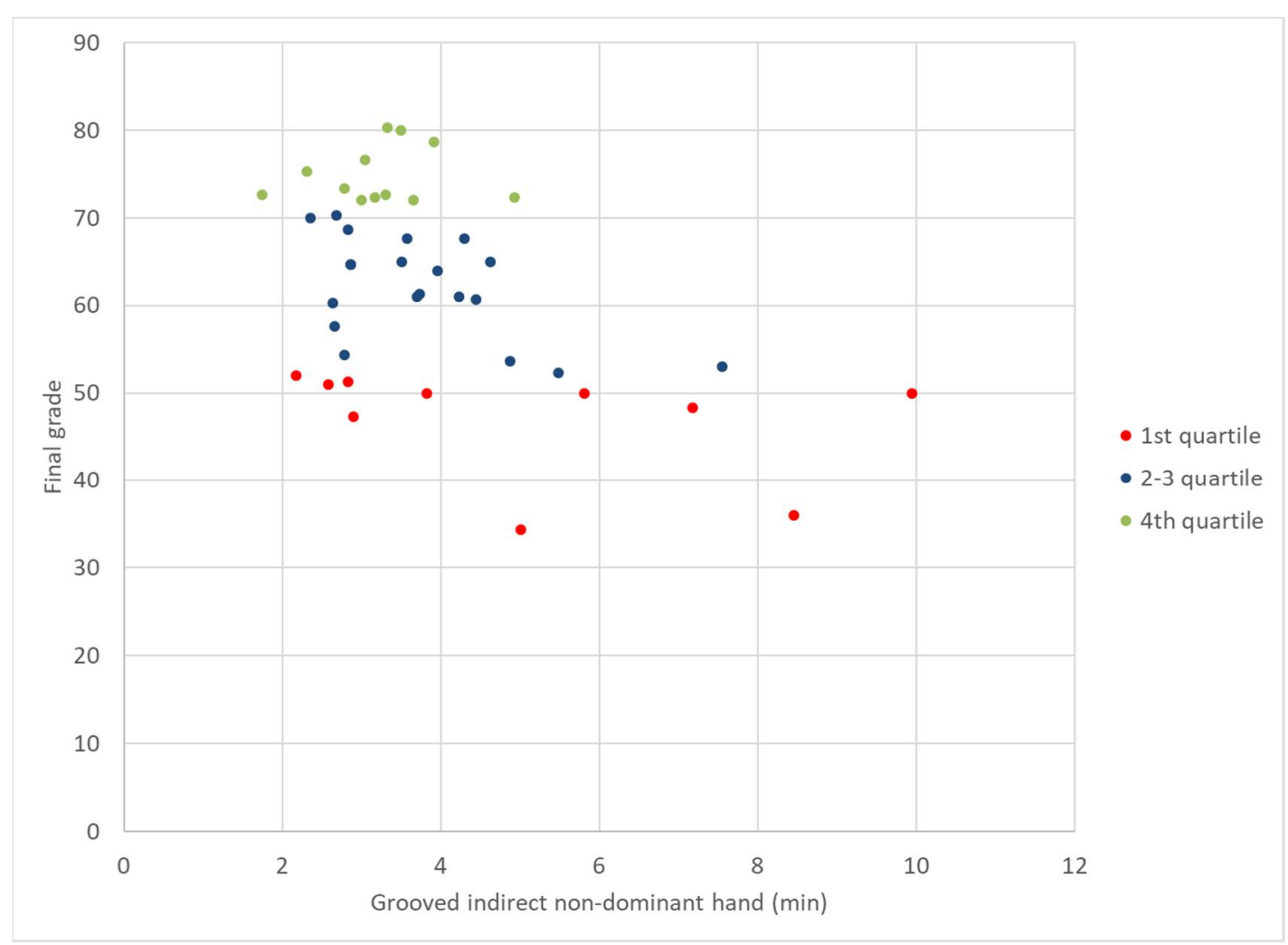

Figure 3. Students' final course grades in quartiles as a function of Grooved indirect non-dominant hand scores.

\section{Discussion}

Fourth year dentistry students at Tel Aviv University undergo practical training in the phantom lab in order to acquire the manual skills required for treating patients. The prosthodontics course constitutes a threshold condition for continuing to the fifth year, 
which concentrates on treating patients. A considerable proportion of students fail to acquire manual skills in the pre-clinical phantom course or pass with a relatively low grade. Our overall aim was to increase the success rate in the pre-clinical prosthodontics course.

The development of the novel portable tool simulated the principles of the Grooved Pegboard test but was modified to a pseudo-patient's mouth (phantom-like) for the purpose of home training of dentistry students in basic skills required in the prosthodontics phantom course. The rationale for the training was based on the transfer of learning theory. Accordingly, repeated training of one motoric task leads to significant improvement in the performance of another motoric task that has similar components [16]. The extent of transfer of training between one learning task and another task has long been recognized as depending on the similarity of the tasks [22-25]. Therefore, to assure a high degree of transfer from a motoric activity learned in the portable tool to motoric tasks required in the phantom course, we designed the tasks performed on the portable tool to closely resemble the phantom tasks. Examples are the insertion of small pins $(1 \mathrm{~mm}$ diameter and $10 \mathrm{~mm}$ length) into holes on the tooth at a 6-degree tilt to the tooth's length axis, which corresponds to the correct preparation of a tooth and the use of tweezers and dental mirrors. Moreover, the tasks in both settings were carried out on similar artificial jaws. In this study, we identified a precise model for predicting student success with $86 \%$ accuracy, according to students' manual scores obtained before the prosthodontic course: the Grooved Pegboard test with dominant hand under direct vision, the Grooved Pegboard test with non-dominant hand under direct vision, the Grooved Pegboard test with non-dominant hand under indirect vision, and PhantHome under direct vision. However, the logistic regression analysis using the Enter method showed that Grooved Pegboard Test_Indirect_Non Dominanat and PhantHome_Direct hand provide adequate information for predicting the success in prosthodontic course. Moreover, classifying the final grades of the course and relating the results to the students scored in Grooved Pegboard Test_Indirect_Non Dominanat (Figure 3) shows that this test is sensitive in addressing the weak students. This test does not involve dental models (as the PhantHome model), and test duration is short and can serve as an additional tool in admission test for dental school.

This indicates that students who showed high manual ability on the motor tests performed before the prosthodontics course maintained better performance in relation to other students at the end of the course, meaning that the manual skill that is required in dentistry entails, to some degree, an innate ability. Innate fine motor skills and manual ability contribute to success in the phantom course, and a student with less manual capabilities would require more guidance and intensive training to pass the course. This may explain why some dentists feel frustrated during their clinical practice and leave the dental profession for professions that are more appropriate for them. Hence, the identification of innately less manually capable candidates and students, who could benefit from more intensive training before reaching clinical phantom courses, is of high importance.

In our study, it was found that there was greater difficulty in performing the motor tasks in indirect vision compared to the same tasks in direct vision. The performance level decreased (time needed to insert pins) by 3.5 times and 2.5 times in the transition between the direct tasks to the indirect task in the Grooved test at T0 and T1, respectively. Additionally, in the PhantHome test, the performance level dropped 1.5 times at both T0 and T1. Despite a significant improvement in all tests with indirect vision at T1, after a month's training in the PhantHome tool, the difficulty of performing indirect vision tasks was still a challenge. These results are consistent with other studies demonstrating that indirect vision is dependent on students' perceptual ability and orientation [26], but is significantly improved after appropriate training [27-29].

Interestingly, training in the PhantHome tool for one month significantly improved not only the performance in the PhantHome portable tool but also in the Grooved tests, requiring the same skills of orientation and spatial perception that the PhantHome's tasks required. This finding is consistent with the results of similar studies in which the effect of the additional training similar to the requested task was assessed on the performance 
of this task [30-33]. These studies have shown that there is a significant improvement as a result of the additional training. The conclusion reached by the researchers is that the required duration of training can be shortened, and similar results can be reached by training in a simpler, yet similar, task $[34,35]$

In our study, we found a significant and inverse relationship between the various motor tests and the final practical grade in the prosthodontic course. It was interesting to find that the two of the strongest correlations found for the final practical grade in the prosthodontics course were by conducting the Grooved test under indirect vision by the non-dominant hand and the PhantHome test in direct vision with the dominant hand. In the study by Strenge et al. [36], a significant correlation was found between the Grooved test performed in the non-dominant hand and the test that examines the concentration level of medical students. These researchers concluded that in order for the Grooved test to be performed with the non-dominant hand, maximum and greater concentration is needed in comparison with the test involving the dominant hand [37-41]. Our results support these findings (Figure 3). It has to be emphasized that performing the PhantHome test at $\mathrm{T} 0$ challenges the novice students, as they never before performed any task in the oral cavity. Although this test might not be applied by institutions as part of the admission requirements because applicants never coped in oral cavity, the Grooved Peg Board test might achieve similar manual capabilities classifications, specifically with the non-dominant hand, which is regularly used during any dental treatment (e.g., holding and directing the dental mirror, soft tissue retraction). We can postulate that the tests performed in a prosthodontic also require a large concentration to be successful, much like the dental procedures required in dentistry. Hence, it is possible that the reason for the strongest relationship observed for the final practical grade in the course by the nondominant Grooved test is the similarity in terms of the level of concentration required to complete the tasks.

\section{Conclusions}

1. The ability for orientation and spatial perception required in a prosthodontics course is governed by a combination of acquired ability that improves significantly after training and innate ability.

2. There is a positive transfer in learning: PhantHome training led to improved performance on the Grooved tests without further training on these tests. Therefore, training in the PhantHome tool can significantly improve performance in the prosthodontics phantom course.

3. A Prediction model was found using the scores of the various motor tests at T0, which predicted success in a prosthodontics course with $86 \%$ accuracy.

Author Contributions: Conceptualization, D.L. and T.B.; methodology, T.B. and D.L.; software, R.P.; validation, G.B.-I., A.S. and E.D.; formal analysis, S.M.; investigation, D.L.; resources, A.S.; data curation, R.P.; writing — original draft preparation, A.S. and G.B.-I;; writing—review and editing, A.S. and G.B.-I.; visualization, S.M.; supervision, R.P. and T.B.; project administration, A.S.; funding acquisition, A.S. and G.B.-I. All authors have read and agreed to the published version of the manuscript.

Funding: This research received no external funding.

Institutional Review Board Statement: The study was conducted according to the guidelines of the Declaration of Helsinki and approved by the Institutional Review Board (or Ethics Committee) of Tel Aviv University.

Informed Consent Statement: Informed consent was obtained from all subjects involved in the study.

Data Availability Statement: Data available on request due to restrictions, e.g., privacy or ethical.

Conflicts of Interest: The authors declare no conflict of interest. 


\section{Appendix A}

\begin{tabular}{cc}
\hline Abbreviations & Meaning \\
\hline Pegboard_Direct_Dominant Hand & Performing the Grooved Pegboard test by direct vision using dominant hand \\
\hline $\begin{array}{c}\text { Grooved Pegboard } \\
\text { _Direct_Non Dominant Hand } \\
\text { Grooved Pegboard } \\
\text { indirect dominant hand } \\
\begin{array}{c}\text { Grooved Pegboard } \\
\text { indirect Non dominant hand }\end{array}\end{array}$ & Performing the Grooved Pegboard test by direct vision using non-dominant hand \\
\hline FPD & Performing the Grooved Pegboard test by indirect vision using non-dominant hand \\
\hline Final.Phantom & Fixed partial denture \\
\hline PhantHome_Direct & The final phantom grade \\
\hline PhantHome_Indirect & Performing the PhantHome test in direct vision \\
\hline
\end{tabular}

\section{References}

1. Luck, O.; Reitemeier, B.; Scheuch, K. Testing of fine motor skills in dental students. Eur. J. Dental Educ. 2000, 4, 10-14. [CrossRef]

2. Suddick, R.P.; Yancey, J.M.; Wilson, S. Mirror-tracing and Embedded Figures tests as predictors of dental students' performance. J. Dental Educ. 1983, 47, 149-154. [CrossRef] [PubMed]

3. Shillingburg, H.T. Fundamentals of Fixed Prosthodontics, Textbook, 4th ed.; Quintessence Publishing: Berlin, Germany, $2012 ;$ p. 131.

4. Potts, R.G.; Shililingburg, H.T.; Ducanson, M.G. Retention and resistance of preparation for cast restoration. J. Prosthet. Dent. 1980, 43, 303-308. [CrossRef]

5. Schwartz, I.S. A review of methods and techniques to improve the fit of cast restorations. J. Prosthet. Dent. 1986, 56, 279-283. [CrossRef]

6. Kaufman, E.G.; Coelho, D.H.; Colin, L. Factors influencing the retention of cemented gold casting. J. Prosthet. Dent. 1961, 11, 487. [CrossRef]

7. Jorgensen, K.D. The relationship between retention and convergence angle in cemented veneer crowns. Acta Odontol. Scand. 1955, 13, 35-40.

8. Weed, R.M.; Suddick, R.P.; Kleffner, J.H. Taper of clinical and typodonts crown prepared by dental students. J. Dental Res. 1984, 63, 286.

9. Noonan, J.E., Jr.; Goldfogel, M.H. Converge of the axial walls of full veneer crown preparation in dental school environment. J. Prosthet. Dent. 1991, 66, 706-708. [CrossRef]

10. Callan, R.S.; Palladino, C.L.; Furness, A.R.; Bundy, E.L.; Ange, B.L. Effectiveness and feasibility of utilizing E4D technology as a teaching tool in a preclinical dental education environment. J. Dental Educ. 2014, 78, 1416-1423. [CrossRef]

11. Callan, R.S.; Blalock, J.S.; Cooper, J.R.; Coleman, J.F.; Looney, S.W. Reliability of CAD/CAM technology in assessing crown preparations in a preclinical dental school environment. J. Dental Educ. 2014, 78, 40-50. [CrossRef]

12. Carly, F.; Justin, M.; Yasushi, T.; Raina, C.; Lisa, L.; Cliff, L.; John, D.; Shigemi, I.N. Dental Students' perceptions of digital assessment software for preclinical tooth preparation exercises. J. Dental Educ. 2016, 81, 597-603.

13. Ben Gal, G.; Weiss, E.I.; Gafni, N.; Ziv, A. Preliminary assessment of faculty and student perception of a haptic virtual reality simulator for training dental manual dexterity. J. Dental Educ. 2011, 75, 496-504.

14. Jasinevicius, T.R.; Landers, M.; Nelson, S.; Urbankova, A. An evaluation of two dental simulation systems: Virtual reality versus contemporary non-computer assisted. J. Dental Educ. 2004, 68, 1151-1162. [CrossRef]

15. Gottlieb, R.; Lanning, S.K.; Gunsolley, J.C.; Buchanan, J.A. Faculty impressions of dental students' performance with and without virtual reality simulation. J. Dental Educ. 2011, 75, 1443-1451. [CrossRef]

16. Thorndike, E.L. The effect of practice in the case of purely intellectual function. Am. J. Psychiatr. 1908, 19, 374-384. [CrossRef]

17. Neuman, L.M. A simple exercise for teaching mirror vision skills. J. Dental Educ. 1988, 52, 170-172. [CrossRef]

18. Gunter, M.R.; Anne, K. Training device for dental students to practice mirror- inverted movement. J. Dental Educ. 2011, 75, $1280-1284$.

19. Bohnen, N.I.; Kuwabara, H.; Constantine, G.M.; Mathis, C.A.; Moore, R.Y. Grooved Pegboard Test as a biomarker of nigrostriatal denervation in Parkinson's disease. Neurosci. Lett. 2007, 424, 185-189. [CrossRef]

20. Bryden, P.J.; Roy, E.A. A new method of administering the Grooved Pegboard Test: Performance as a function of handedness and sex. Brain Cogn. 2005, 58, 258-268. [CrossRef] [PubMed]

21. Strenge, H.; Niederberger, U. Unidirectional interference in use of nondominant hand during concurrent Grooved Pegboard and random number genera-tion tasks. Percept. Mot. Skills 2008, 106, 763-774. [CrossRef] [PubMed]

22. Kao, E.; Ngan, P.; Wilson, S.; Kunovich, R. Wire-bending test as a predictor of preclinical performance by dental students. Percept. Mot. Skills 1990, 71, 667-673. [CrossRef] 
23. Kothe, C.; Hissbach, J.; Hampe, W. Prediction of practical performance in preclinical laboratory courses: The return of wire bending for admission of dental students in Hamburg. GMS Z. Med. Ausbild. 2014, 31, 22.

24. Lugassy, D.; Levanon, Y.; Shpack, N.; Levartovsky, S.; Pilo, R.; Brosh, T. An interventional study for improving the manual dexterity of dentistry students. PLoS ONE 2019, 14, e0211639. [CrossRef] [PubMed]

25. Osgood, C.E. The similarity paradox in human learning: A resolution. Psychol. Rev. 1949, 56, 132-143. [CrossRef] [PubMed]

26. Muscio, B. Motor capacity with special reference to vocational guidance. Br. J. Psychol. Gen. Sect. 1922, 13, 157-184. [CrossRef]

27. Oliver, E.C. A sensorimotor program for improving writing readiness skills in elementary age children. Am. J. Occup. Ther. 1989, 44, 111-115. [CrossRef]

28. Smith, J.C. Effects of occupational therapy services on fine motor and functional performance in preschool children. Am. J. Occup. Ther. 2000, 54, 372-380. [CrossRef]

29. Lugassy, D.; Levanon, Y.; Pilo, R.; Shely, A.; Rosen, G.; Meirowitch, A.; Brosh, T. Predicting the clinical performance of dental students with a manual dexterity test. PLoS ONE 2018, 13, e0193980. [CrossRef]

30. Schwibbe, A.; Kothe, C.; Hampe, W.; Konradt, U. Acquisition of dental skills in preclinical technique course: Influence of spatial and manual abilities. Adv. Health Sci. Educ. 2016, 21, 841-857. [CrossRef]

31. Willis, D.O.; Kincheloe, J.E. Teaching dental students mirror vision skills. J. Dental Educ. 1983, 47, 311-316. [CrossRef]

32. Boyd, M.A.; Rucker, L.M. Effects of immediate introduction of indirect vision on performance and posture. J. Dental Educ. 1987, 51, 98-101. [CrossRef]

33. McClure, A.R.; Roomian, T.C.; Eisen, S.E.; Kugel, G.; Amato, R.B. Jumpstart Mirror Trainer: A New Device for Teaching Mirror Skills to First-Year Dental Students. J. Dental Educ. 2019, 83, 1199-1204. [CrossRef]

34. Gagne, R.M.; Foster, H. Transfer of training from practice on components in a motor skill. J. Exp. Psychol. 1949, 39, 47-68. [CrossRef]

35. Bezdicek, O.; Nikolai, T.; Hoskovcova, M.; Stochl, J.; Brožová, H.; Dušek, P.; Zárubová, K.; Jech, R.; Rưžička, E. Grooved pegboard predicates more of cognitive than motor involvement in parkinson's disease. Assessment 2014, 21, 723-730. [CrossRef]

36. Strenge, H.; Niederberger, U.; Seelhorst, U. Correlation between tests of attention and performance on grooved and Purdue pegboard in normal subjects. Percept. Mot. Skills 2002, 95, 507-514. [CrossRef]

37. Spratley, M.H. Regression analysis of dexterity tests and dental students' practical examination results. Aust. Dental J. 1992, 37, 461-466. [CrossRef] [PubMed]

38. Stacey, D.G.; Whittaker, J.M. Predicting academic performance and clinical competency for international dental students: Seeking the most effective measures. J. Dental Educ. 2005, 69, 270-280. [CrossRef] [PubMed]

39. Spratley, M.H. Aptitude testing and the selection of dental students. Aust. Dental J. 1990, 35, 159-168. [CrossRef]

40. Kunovich, R.; Rachid, R.G. Mirror training in three dimensions for dental students. Percept. Mot. Skills 1992, 75, 923-928. [CrossRef] [PubMed]

41. Nishida, M.; Sohmura, T.; Takahashi, J. Training in tooth preparation utilizing a support system. J. Oral Rehabil. 2004, 31, 149-154. [CrossRef] [PubMed] 